This item was submitted to Loughborough's Research Repository by the author.

Items in Figshare are protected by copyright, with all rights reserved, unless otherwise indicated.

\title{
Formation of near-IR excitons in low-dimensional CuSbS2
}

PLEASE CITE THE PUBLISHED VERSION

https://doi.org/10.1021/acs.jpcc.1c06530

PUBLISHER

American Chemical Society (ACS)

VERSION

VoR (Version of Record)

\section{PUBLISHER STATEMENT}

This is an Open Access Article. It is published by American Chemical Society under the Creative Commons Attribution 4.0 Unported Licence (CC BY). Full details of this licence are available at: http://creativecommons.org/licenses/by/4.0/

\section{LICENCE}

CC BY 4.0

\section{REPOSITORY RECORD}

Conley, Kevin M, Caterina Cocchi, and Tapio Ala-Nissila. 2021. "Formation of Near-ir Excitons in Lowdimensional Cusbs2". Loughborough University. https://hdl.handle.net/2134/16883596.v1. 


\title{
Formation of Near-IR Excitons in Low-Dimensional CuSbS
}

\author{
Kevin M. Conley, Caterina Cocchi, and Tapio Ala-Nissila*
}

Cite This: J. Phys. Chem. C 2021, 125, 21087-21092

Read Online

ABSTRACT: The electronic and optical properties of low-dimensional semiconductors are typically quite different from those of their bulk counterparts. Yet, the optical gap of two-dimensional copper antimony disulfide $\left(\mathrm{CuSbS}_{2}\right)$ does not dramatically change with decreasing thickness of the material. The absorption onset remains at about $1.5 \mathrm{eV}$ in the monolayer, bilayer, and bulk materials. Using density functional theory and many-body perturbation theory, we rationalize this behavior through the interplay of quantum confinement, electron-hole interactions, and the formation of surface states. Specifically, the spatial confinement in thin layers induces strongly bound optical transitions in the near-infrared region. Our results explain the optical properties in copper antimony disulfide platelets of varying thickness and set these materials as potential candidates for novel photovoltaic devices and near-infrared sensors.

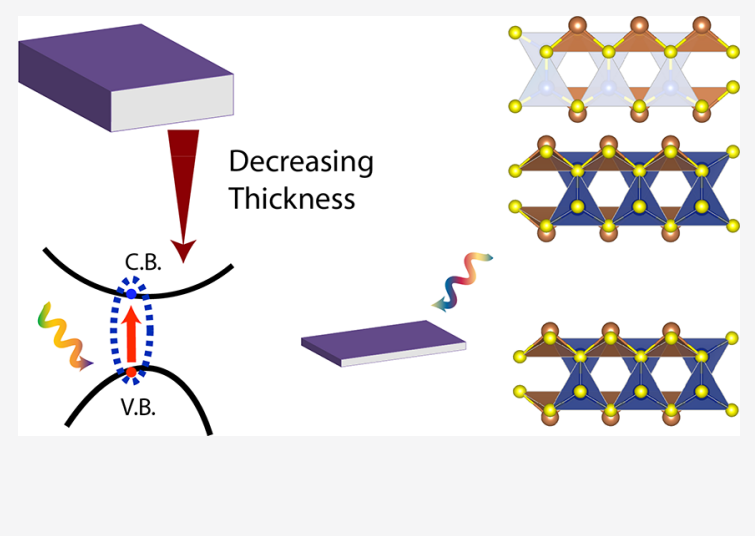

\section{INTRODUCTION}

Thin-film materials for solar photovoltaics are challenging traditional wafer silicon in the renewable energy sector. ${ }^{1-5}$ Scarcity and competing industrial demands for the constituent elements of CdTe and copper indium gallium disulfide/ diselenide devices have driven the search for alternative systems with comparable performance. ${ }^{5-7}$ Among them, $\mathrm{CuSbS}_{2}$ is a promising material formed by earth-abundant elements and characterized by a large absorption coefficient at relevant frequencies for solar light harvesting. ${ }^{8,9}$ The optical gap of bulk $\mathrm{CuSbS}_{2}$ ranges between 1.3 and $1.5 \mathrm{eV}$.,10-13 Theoretical studies based on quantum density functional theory (DFT) calculations ${ }^{14,15}$ have shown that in this material the fundamental gap is indirect, like in bulk silicon, and that the direct gap appears at a marginally higher energy of 1.73 eV. ${ }^{16-20}$

Recently, single-crystal $\mathrm{CuSbS}_{2}$ platelets of varying thickness have been experimentally prepared using the hot injection method. ${ }^{21,22}$ This bottom-up approach enables the synthesis of nanomaterials with well-defined size and homogeneous shape down to monolayer thickness. ${ }^{22}$ Interestingly, in $\mathrm{CuSbS}_{2}$, quantum confinement effects accompanying the nanostructuring process do not lead to a sizable increase of the optical gap, as typically observed in low-dimensional semiconductors and insulators in comparison with their bulk counterparts. ${ }^{23-27}$ For example, in transition metal dichalcogenides, spatial confinement increases absorption and photoluminescence ${ }^{28-34}$ or even induces superconductivity. ${ }^{35}$ Enhancement of optical activities has also been observed in hexagonal boron nitride $^{36-38}$ and heterostructures thereof, ${ }^{39,40}$ as well as in the more recently produced silicene. ${ }^{41-44}$
Such peculiar behavior of $\mathrm{CuSbS}_{2}$ has already been explored in a DFT study, ${ }^{45}$ which, however, has not been able to convincingly rationalize the change of the optical gap of thin platelets $(1-5 \mathrm{~nm})$ of this material compared to the bulk. Understanding the fundamental mechanism that is responsible for this counterintuitive behavior is essential to fully exploit the potential of $\mathrm{CuSbS}_{2}$ in optoelectronic and photovoltaic applications. Most importantly, it is mandatory to gain further insight into exotic behaviors of quantum-confined semiconductors.

In this work, based on DFT and many-body perturbation theory (MBPT), ${ }^{46}$ we rationalize why the optical gap of twodimensional $\mathrm{CuSbS}_{2}$ platelets does not increase compared to their bulk counterparts. From the analysis of the band structure, the density of states, the charge-density distribution, and finally the dielectric and optical response of this material in the monolayer, bilayer, and bulk form, we ascribe this behavior to the concurrent contribution of localized surface states that are formed in the nanoconfined systems and strongly bound excitons that appear therein.

\section{METHODS}

All calculations presented in this work are performed with the Vienna $a b$ initio simulation package (VASP) ${ }^{47}$ utilizing the projector-augmented wave (PAW) method. ${ }^{48,49}$ The electronic

Received: August 10, 2021

Published: September 20, 2021 
a)

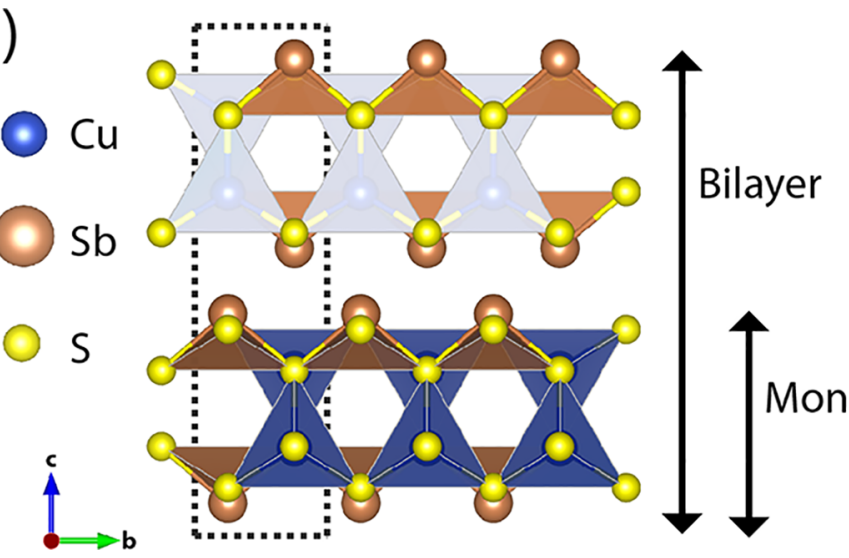

b)

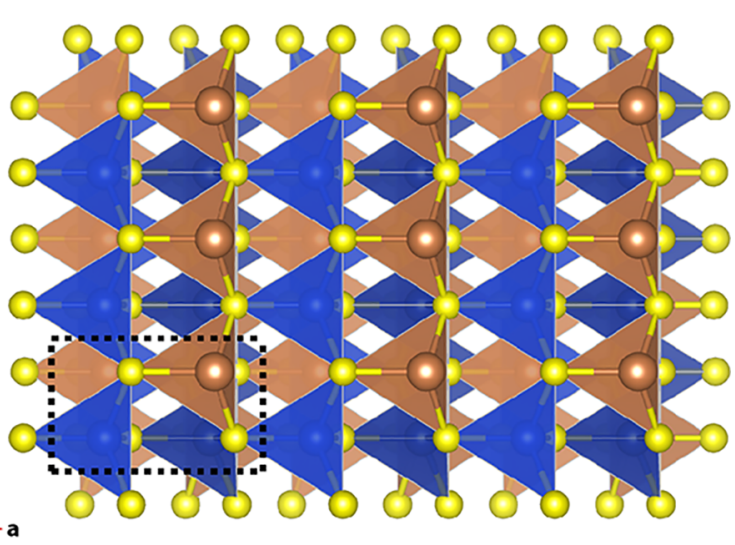

Figure 1. (a) Layered and (b) bulk $\mathrm{CuSbS}_{2}$ with $\mathrm{Cu}, \mathrm{Sb}$, and $\mathrm{S}$ atoms shown as blue, brown, and yellow spheres.

structure was calculated using DFT with the Heyd-ScuseriaErnzerhof (HSE06) hybrid functional. ${ }^{50}$ A $4 \times 6 \times 2 k$-point mesh and $370 \mathrm{eV}$ cutoff are used, and the structures were assumed to be relaxed when the forces on each atom were smaller than $10 \mathrm{meV}^{-1}$. The mono- and bilayer structures are simulated including in the unit cell $9 \AA$ of vacuum along the (001) surface in order to prevent unphysical interactions between the replicas. We checked this value by ensuring the band gap remained constant with an additional vacuum and by checking that the total charge density decays to less than $1 \times$ $10^{-6} a_{0}^{-3}$ in the nonperiodic direction.

The quasiparticle band structures are calculated with a single-shot $\mathrm{G}_{0} \mathrm{~W}_{0}$ approach applied on top of the HSE06 electronic structure. The GW band structure converged with 600 bands or 320 bands in the case of the monolayer. The optical properties were calculated by solving the BetheSalpeter equation (BSE) $)^{51}$ and included contributions beyond the Tamm-Dancoff approximation. ${ }^{52}$ The 30 highest valence and the 60 lowest conduction bands are considered in the bilayer and bulk, and the 28 highest valence and the 60 lowest conduction bands are considered in the monolayer. Spurious contributions arising from the vacuum in the layered structures are excluded by rescaling the dielectric permittivity with the effective volume of the layer.

\section{RESULTS AND DISCUSSION}

The optimized lattice constants and atomic positions obtained for bulk $\mathrm{CuSbS}_{2}$ (Figure 1) are consistent with previous findings in the literature obtained on similar footing ${ }^{16,17}$ (see Table $\mathrm{S} 1$ in the Supporting Information). $\mathrm{CuSbS}_{2}$ is an orthorhombic crystal with space group Pnma. The space groups of the bilayer and monolayer are $\mathrm{P} 2_{1} / m$ and $P m$, respectively. In the layered crystals, no surface reconstruction is found, although the atoms are bound closer together normal to the (001) surface. Specifically, the material volume in the monolayer shrinks by $4.2 \%$ compared to the bulk, as an effect of the quantum confinement along the $c$ axis (see Figure 1a), in agreement with experimental findings on powder $\mathrm{CuSbS}_{2}$ platelets. $^{22}$ In the bilayer, this reduction is only $0.8 \%$.

From the analysis of the electronic properties, we find for bulk $\mathrm{CuSbS}_{2}$ an indirect fundamental gap of $1.67 \mathrm{eV}$, as predicted from DFT using HSE06 (see Figure 2a), in agreement with previous calculations. ${ }^{16,17,45}$ Despite quantum confinement, both monolayer and bilayer $\mathrm{CuSbS}_{2}$ exhibit smaller band gaps than the bulk, with the former featuring the narrowest gap. The inclusion of the quasi-particle correction
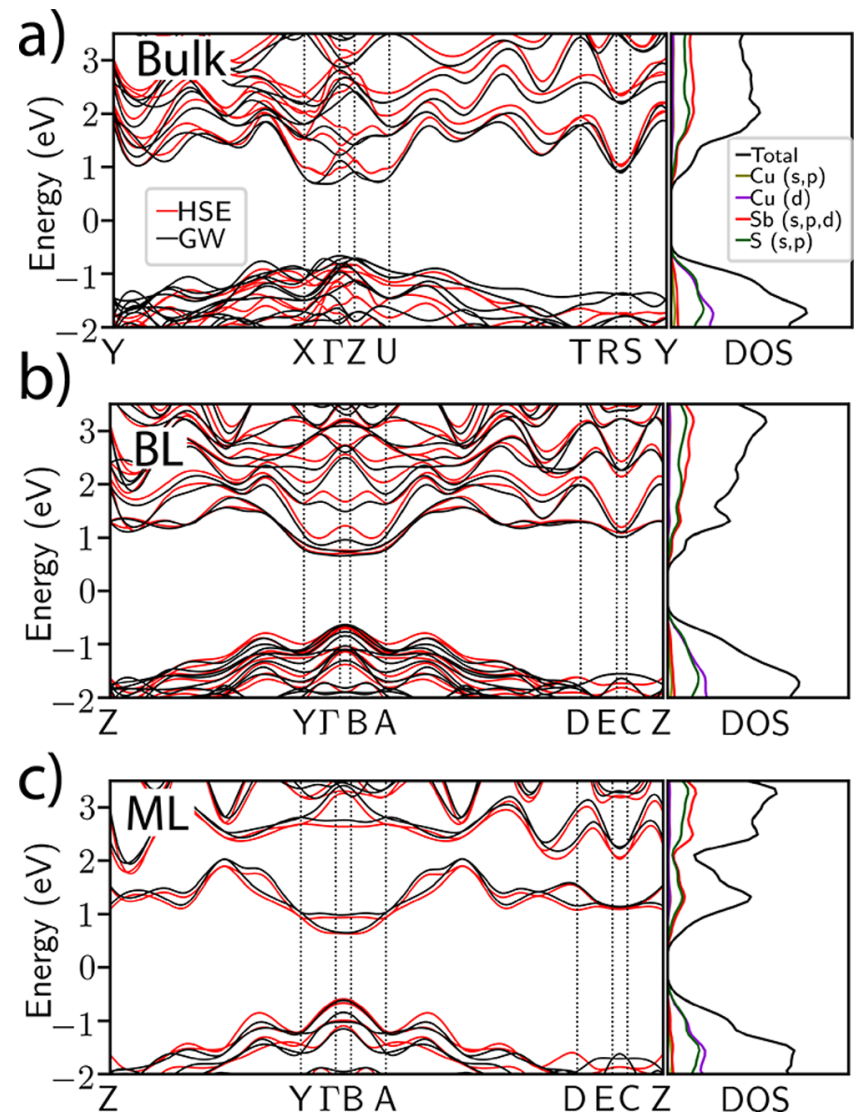

Figure 2. GW (black lines) and HSE06 band structure (red lines) of (a) bulk, (b) bilayer (BL), and (c) monolayer (ML) $\mathrm{CuSbS}_{2}$. The density of states and atom-projected contributions calculated using HSE06 are shown adjacent to the band structures.

via the perturbative $\mathrm{G}_{0} \mathrm{~W}_{0}$ approach applied on top of the HSE06 electronic structure leads to a reduction of the band gap in both the bulk and bilayer and to an increase in the monolayer. The nature of the gap (indirect in the bulk and direct in the low-dimensional sheets) is unaffected by the inclusion of the self-energy contribution (see Table 1).

The crossover from indirect- to direct-band-gap material going from bulk to the mono- and bilayer is ubiquitous in layered semiconductors. ${ }^{28,33,53,54}$ In order to understand this behavior in $\mathrm{CuSbS}_{2}$, we analyze the band dispersion shown in Figure 2. The Brillouin zone of each system is shown in Figure 
Table 1. Band Gaps and Optical Transitions of Bulk and Layered $\mathrm{CuSbS}_{2}$

\begin{tabular}{|c|c|c|c|c|}
\hline & $\begin{array}{l}\text { indirect band } \\
\text { gap }(\mathrm{eV})\end{array}$ & $\begin{array}{l}\text { direct band } \\
\text { gap }(\mathrm{eV})\end{array}$ & $\begin{array}{c}E_{\text {optical }} \\
(\mathrm{eV})\end{array}$ & $\begin{array}{l}E_{\mathrm{b}} \\
(\mathrm{eV})\end{array}$ \\
\hline \multicolumn{5}{|l|}{ Bulk } \\
\hline this work, MBPT & 1.35 & 1.40 & 1.20 & 0.20 \\
\hline this work, HSE06 & 1.67 & 1.71 & & \\
\hline $\mathrm{HSE} 6^{16}$ & 1.68 & 1.73 & & \\
\hline $\mathrm{HSE}^{17} 6^{17}$ & 1.69 & & & \\
\hline \multicolumn{5}{|l|}{ Layered } \\
\hline this work, MBPT BL & & 1.30 & 0.95 & 0.35 \\
\hline this work, MBPT ML & & 1.30 & 0.76 & 0.54 \\
\hline this work, HSE06 BL & & 1.38 & & \\
\hline $\begin{array}{l}\text { this work, HSE06 } \\
\text { ML }\end{array}$ & & 1.25 & & \\
\hline \multicolumn{5}{|l|}{ Experimental } \\
\hline $\begin{array}{l}\text { platelets }(25-50 \mathrm{~nm} \\
\text { thick })^{22}\end{array}$ & $1.51^{a}$ & $1.57^{a}$ & $0.92^{b}$ & $0.65^{c}$ \\
\hline $\begin{array}{l}\text { platelets }(1-5 \mathrm{~nm} \\
\text { thick })^{22}\end{array}$ & $1.44^{a}$ & $1.52^{a}$ & $1.04^{b}$ & $0.48^{c}$ \\
\hline \multicolumn{5}{|c|}{$\begin{array}{l}{ }^{a} \text { Optical gap measured using diffuse reflectance spectroscopy. } \\
b^{b} \text { Energies less than } 0.85 \mathrm{eV} \text { were beyond the experimental range. } \\
{ }^{c} \text { The experimental binding energy, } E_{\mathrm{b}} \text {, was obtained by subtracting } \\
\text { the optical transition energy from the direct optical gap. }\end{array}$} \\
\hline
\end{tabular}

S1. In the bulk material, the valence band maximum of bulk $\mathrm{CuSbS}_{2}$ lies between the $\Gamma$ and $Z$ points, and the conduction band minimum lies between the $\Gamma$ and $X$ points. The smallest, direct gap lies between the $\Gamma$ and $X$ points. Interestingly, in the layered materials, the highest valence band and the lowest conduction bands flatten along the $B$ and $Y$ directions. This change in the band dispersion is responsible for the crossover to a direct band gap. The variation in the fundamental band gap in increasingly confined layers is attributed to the reduced band dispersion accompanied by the formation of a surface state at the Sb-terminated (001) surface. The states have $\mathrm{Cu} d$ and $S \mathrm{sp}$ character at the top of the valence band and $S \mathrm{sp}$ and $\mathrm{Sb}$ spd character at the bottom of the conduction band (Figure 2). There is no significant change in the character of the states in the layered materials. The partial DOS of bulk $\mathrm{CuSbS}_{2}$ is consistent with previous DFT studies in bulk $\mathrm{CuSbS}_{2} .{ }^{20}$

To confirm the presence of surface states in the $\mathrm{Sb}$ terminated dangling surface of mono- and bilayer $\mathrm{CuSbS}_{2}$, we inspect the electron density at the conduction band minimum. The results plotted along a two-dimensional slice in the (001) plane (see Figure 3) confirm that the electron density accumulates at the surface of the low-dimensional structures. The electron density between the layers in the bilayer is similar to the interlayer electron density in the bulk material. The electron density spills out of the monolayer more than the bilayer (Figure 3c). This results in electron density accumulation between the adjacent surface $\mathrm{Sb}$ atoms.

Having clarified the electronic characteristics of lowdimensional $\mathrm{CuSbS}_{2}$, we complete our analysis by inspecting its optical properties, calculated by solving the $\mathrm{BSE}^{51}$ of MBPT. Bright excitations below the fundamental band gap occur at $1.20,0.95$, and $0.76 \mathrm{eV}$ for the bulk material, bilayer, and monolayer, respectively, as a signature of excitonic effects (see Figure 4 as well as Figure S2 for the real part and the out-

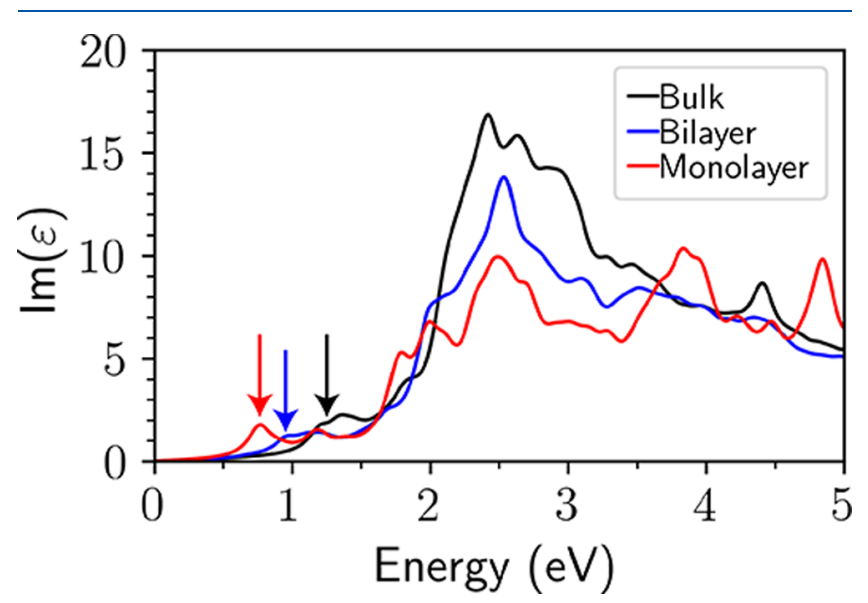

Figure 4. Imaginary component of the dielectric permittivity of bulk, bilayer, and monolayer $\mathrm{CuSbS}_{2}$ in the in-plane directions calculated using GW+BSE. The lowest-energy optical transition in each structure is marked with an arrow.

of-plane components). The contributions of the BSE eigenstates to each exciton are represented as a fat band plot in reciprocal space (Figure S3). The contributions between the direct electron-hole pairs are predominately from the bands near the band gap. The strength of the contributions is more in the layered materials, which have a reduced band dispersion. The exciton binding energy, calculated as $E_{\mathrm{b}}=E_{\mathrm{g}}^{\mathrm{GW}}-E_{\text {optical }}$, is $0.20 \mathrm{eV}$ in the bulk where $E_{\mathrm{g}}^{\mathrm{GW}}$ is the direct gap from $\mathrm{GW}$ and $E_{\text {optical }}$ is the lowest-energy excitation from BSE. The reduced

\section{0}

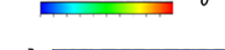

a)
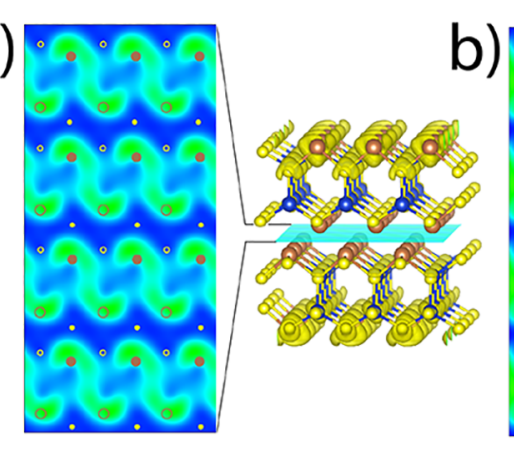
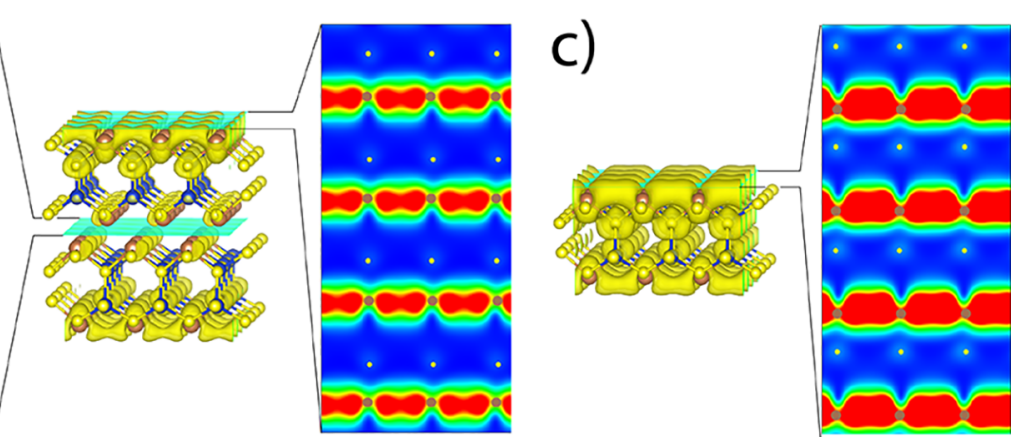

Figure 3. Spatial distribution of the probability density associated with the conduction band minimum of (a) bulk, (b) bilayer, and (c) monolayer $\mathrm{CuSbS}_{2}$ calculated from DFT. The displayed isosurface value is $0.003 a_{0}^{-3}$. The positions of atoms above (below) the considered plane are marked by open (closed) circles: brown for $\mathrm{Sb}$ and yellow for $\mathrm{S}$. 


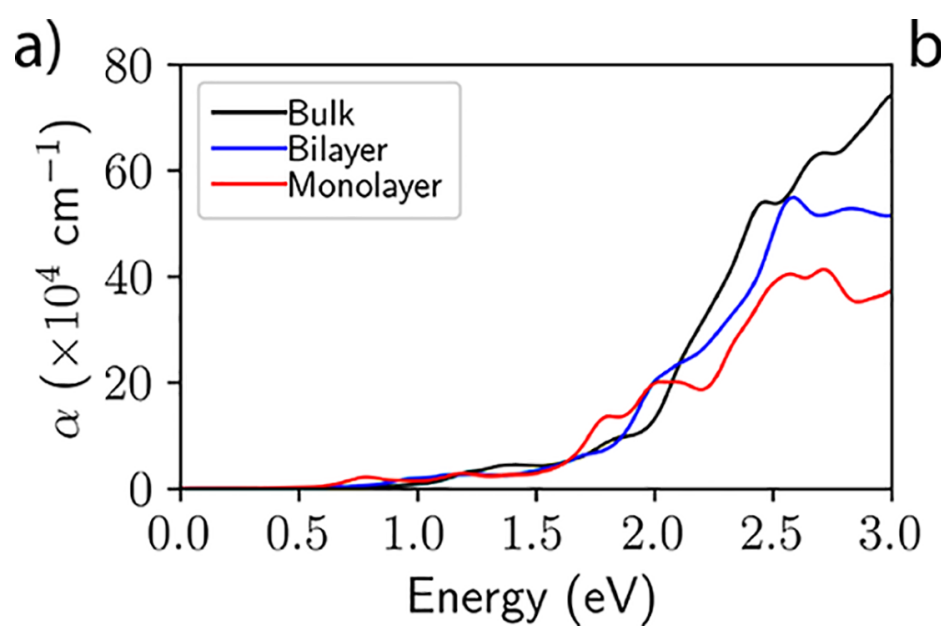

b)

Figure 5. (a) Absorption coefficient, $\alpha$, and (b) Tauc plot for bulk, bilayer, and monolayer $\mathrm{CuSbS}_{2}$ in the in-plane direction calculated using GW + BSE.

dimensionality of the system increases the exciton binding energy to $0.35 \mathrm{eV}$ in the bilayer and to $0.54 \mathrm{eV}$ in the monolayer (see Table 1). The experimental binding energy is similar to the prediction from theory despite the natural size distribution of platelets in the powder samples. ${ }^{22}$ Furthermore, the effects of ligand passivation, defects, or stacking of monolayers into towers could obscure the band gap crossover and optical properties. ${ }^{55}$ Experimental detection of the changes in the excitonic peaks or lattice would be improved if the ensemble contains only monolayer platelets. We also note that the possible exciton-like peaks in the layered material may be resolved at lower temperatures, which was previously shown for bulk $\mathrm{CuSbS}_{2}{ }^{56}$

For a closer comparison with experimental results, it is convenient to display the optical properties of each material in the form of the absorption coefficient and Tauc plot. The absorption coefficient is $\alpha=4 \pi \kappa / \lambda$, where $\kappa$ is the extinction coefficient and $\lambda$ the wavelength of the incident light. From the plot in Figure $5 \mathrm{a}$, we notice that the absorption coefficient increases above $10^{5} \mathrm{~cm}^{-1}$ between 1.7 and $1.9 \mathrm{eV}$ regardless of dimensionality. In particular, the Tauc plot in Figure $5 \mathrm{~b}$ shows that (i) despite the spatial confinement in the low-dimensional structures the absorption increases steeply at about $1.5 \mathrm{eV}$ in the bulk, bilayer, and monolayer and (ii) weak optical excitations appear below the band gap in all materials, as anticipated in the discussion of the dielectric permittivity (Figure 4). The first finding, (i), is consistent with recent results from diffuse reflectance spectroscopy on platelets which detected only a small decrease $(0.05 \mathrm{eV})$ in the direct optical gap energy in thin ( 1 to $5 \mathrm{~nm}$ ) platelets compared to the thickest platelets $(25-50 \mathrm{~nm}) .^{22}$ The second finding, (ii), is supported by the presence of weak absorption peaks below the optical gap that have been detected in the ellipsometric data of bulk $\mathrm{CuSbS}_{2}{ }^{56}$ and two-dimensional platelets. ${ }^{22}$ The thinnest platelets had a peak at $1.04 \mathrm{eV}$, and energies less than $0.85 \mathrm{eV}$ were beyond the spectral range. ${ }^{22}$

\section{CONCLUSIONS}

In summary, by means of DFT and many-body calculations, we rationalized the peculiar behavior of $\mathrm{CuSbS}_{2}$ which exhibits a reduction of the lowest optical transition going from the bulk to mono- and bilayer films. In addition to the crossover from indirect- to direct-band-gap materials, bilayer and monolayer films of $\mathrm{CuSbS}_{2}$ exhibit (slightly) reduced band gaps compared to their bulk counterpart due to the formation of localized surface states on the dangling facet. On top of this, now in agreement with physical intuition, larger exciton binding energies were found upon increasing quantum confinement, which led to the decrease of the absorption onset going to the bulk, bilayer, and mononlayer, with the latter absorbing radiation in the near-infrared region. By disclosing the fundamental mechanisms behind the peculiar electronic and optical properties of $\mathrm{CuSbS}_{2}$, this study opens important perspectives for nanoengineering this material and related compounds for optoelectronic and photovoltaic applications.

\section{ASSOCIATED CONTENT}

\section{Supporting Information}

The Supporting Information is available free of charge at https://pubs.acs.org/doi/10.1021/acs.jpcc.1c06530.

Relaxed lattice constants and out-of-plane dielectric permittivities for each structure (PDF)

\section{AUTHOR INFORMATION}

\section{Corresponding Author}

Tapio Ala-Nissila - QTF Centre of Excellence, Department of Applied Physics, Aalto University School of Science, FI-00076 Aalto, Finland; Interdisciplinary Centre for Mathematical Modelling, Department of Mathematical Sciences, Loughborough University, Loughborough, Leicestershire LE113TU, United Kingdom; Email: tapio.ala-nissila@ aalto.fi

\section{Authors}

Kevin M. Conley - Department of Chemistry and Materials Science, Aalto University School of Chemical Engineering, FI00076 Aalto, Finland; ㅇo이.org/0000-0001-7780-3096

Caterina Cocchi - Institut für Physik, Carl von Ossietzky Universität Oldenburg, 26129 Oldenburg, Germany; Institut für Physik und IRIS Adlershof, Humboldt-Universität zu Berlin, 12489 Berlin, Germany

Complete contact information is available at: https://pubs.acs.org/10.1021/acs.jpcc.1c06530

\section{Notes}

The authors declare no competing financial interest. 


\section{ACKNOWLEDGMENTS}

This work was performed as part of the Academy of Finland RADDESS project 314488 and QTF Centre of Excellence program (project 312298) (KC, TAN). We acknowledge computational resources provided by the CSC - IT Center for Science (Finland) and by the Aalto Science-IT project (Aalto University School of Science). The work was supported in part by the High Performance Computing Centre Stuttgart (HLRS) and HPC-Europa3. C.C. acknowledges financial support from the German Research Foundation, project number 182087777 (CRC 951), from the German Federal Ministry of Education and Research (Professorinnen programm III), and from the State of Lower Saxony (Professorinnen für Niedersachsen).

\section{REFERENCES}

(1) Walsh, A.; Chen, S.; Wei, S.-H.; Gong, X.-G. Kesterite thin-film solar cells: Advances in materials modelling of $\mathrm{Cu}_{2} \mathrm{ZnSnS}_{4}$. Adv. Energy Mater. 2012, 2, 400-409.

(2) Mitzi, D. B.; Gunawan, O.; Todorov, T. K.; Barkhouse, D. A. R. Prospects and performance limitations for $\mathrm{Cu}-\mathrm{Zn}-\mathrm{Sn}-\mathrm{S}-\mathrm{Se}$ photovoltaic technology. Philos. Trans. R. Soc., A 2013, 371, 20110432.

(3) Pal, K.; Singh, P.; Bhaduri, A.; Thapa, K. B. Current challenges and future prospects for a highly efficient $(>20 \%)$ kesterite CZTS solar cell: A review. Sol. Energy Mater. Sol. Cells 2019, 196, 138-156.

(4) Ramanujam, J.; Bishop, D. M.; Todorov, T. K.; Gunawan, O.; Rath, J.; Nekovei, R.; Artegiani, E.; Romeo, A. Flexible CIGS, CdTe and a-Si: $\mathrm{H}$ based thin film solar cells: A review. Prog. Mater. Sci. 2020, 110, 100619.

(5) Peter, L. M. Towards sustainable photovoltaics: The search for new materials. Philos. Trans. R. Soc., A 2011, 369, 1840-1856.

(6) Fthenakis, V.; Wang, W.; Kim, H. C. Life cycle inventory analysis of the production of metals used in photovoltaics. Renewable Sustainable Energy Rev. 2009, 13, 493-517.

(7) Peccerillo, E.; Durose, K. Copper-antimony and copperbismuth chalcogenides-Research opportunities and review for solar photovoltaics. MRS Energy \& Sustainability 2018, 5, No. E13.

(8) Kumar, M.; Persson, C. $\mathrm{Cu}(\mathrm{Sb}, \mathrm{Bi})(\mathrm{S}, \mathrm{Se})_{2}$ as indium-free absorber material with high optical efficiency. Energy Procedia 2014, 44, 176-183.

(9) de Souza Lucas, F. W.; Peng, H.; Johnston, S.; Dippo, P. C.; Lany, S.; Mascaro, L. H.; Zakutayev, A. Characterization of defects in copper antimony disulfide. J. Mater. Chem. A 2017, 5, 21986-21993.

(10) Rodriguez-Lazcano, Y.; Nair, M.; Nair, P. $\mathrm{CuSbS}_{2}$ thin film formed through annealing chemically deposited $\mathrm{Sb}_{2} \mathrm{~S}_{3}-\mathrm{CuS}$ thin films. J. Cryst. Growth 2001, 223, 399-406.

(11) Kyono, A.; Kimata, M. Crystal structures of chalcostibite $\left(\mathrm{CuSbS}_{2}\right)$ and emplectite $\left(\mathrm{CuBiS}_{2}\right)$ : Structural relationship of stereochemical activity between chalcostibite and emplectite. Am. Mineral. 2005, 90, 162-165.

(12) Zhou, J.; Bian, G.-Q.; Zhu, Q.-Y.; Zhang, Y.; Li, C.-Y.; Dai, J. Solvothermal crystal growth of $\mathrm{CuSbQ}_{2}(\mathrm{Q}=\mathrm{S}, \mathrm{Se})$ and the correlation between macroscopic morphology and microscopic structure. J. Solid State Chem. 2009, 182, 259-264.

(13) Ikeda, S.; Kawaguchi, T.; Koda, Y.; Iiyama, N.; Harada, T.; Nakanishi, S.; Nakatsuka, S.; Nose, Y. Structural and electric properties of $\mathrm{CuSbS}_{2}$ compound semiconductor bulk crystals. Jpn. J. Appl. Phys. 2018, 57, 08RC09.

(14) Hohenberg, P.; Kohn, W. Inhomogeneous Electron Gas. Phys. Rev. 1964, 136, B864-B871.

(15) Kohn, W.; Sham, L. J. Self-Consistent Equations Including Exchange and Correlation Effects. Phys. Rev. 1965, 140, A1133A1138.

(16) Temple, D. J.; Kehoe, A. B.; Allen, J. P.; Watson, G. W.; Scanlon, D. O. Geometry, electronic structure, and bonding in $\mathrm{CuMCh}_{2}(\mathrm{M}=\mathrm{Sb}, \mathrm{Bi} ; \mathrm{Ch}=\mathrm{S}, \mathrm{Se})$ : Alternative solar cell absorber materials? J. Phys. Chem. C 2012, 116, 7334-7340.
(17) Dufton, J. T.; Walsh, A.; Panchmatia, P. M.; Peter, L. M.; Colombara, D.; Islam, M. S. Structural and electronic properties of $\mathrm{CuSbS}_{2}$ and $\mathrm{CuBiS}_{2}$ : Potential absorber materials for thin-film solar cells. Phys. Chem. Chem. Phys. 2012, 14, 7229-7233.

(18) Birkett, M. Optical properties of earth-abundant semiconductors for renewable energy. Ph.D. thesis, University of Liverpool, 2016.

(19) Gassoumi, A.; Musa Saad, M. H.-E.; Alfaify, S.; Ben Nasr, T.; Bouarissa, N. The investigation of crystal structure, elastic and optoelectronic properties of $\mathrm{CuSbS}_{2}$ and $\mathrm{CuBiS}_{2}$ compounds for photovoltaic applications. J. Alloys Compd. 2017, 725, 181-189.

(20) Gupta, G. K.; Chaurasiya, R.; Dixit, A. Thermodynamic stability and optoelectronic properties of $\mathrm{Cu}(\mathrm{Sb} / \mathrm{Bi})(\mathrm{S} / \mathrm{Se})_{2}$ ternary chalcogenides: Promising ultrathin photoabsorber semiconductors. Sol. Energy 2019, 177, 679-689.

(21) Moosakhani, S.; Sabbagh Alvani, A. A.; Mohammadpour, R.; Hannula, P.-M.; Ge, Y.; Hannula, S.-P. Platelet $\mathrm{CuSbS}_{2}$ particles with a suitable conduction band position for solar cell applications. Mater. Lett. 2018, 215, 157-160.

(22) Moosakhani, S.; Sabbagh Alvani, A. A.; Mohammadpour, R.; Ge, Y.; Hannula, S.-P. Solution synthesis of $\mathrm{CuSbS}_{2}$ nanocrystals: A new approach to control shape and size. J. Alloys Compd. 2018, 736, 190-201.

(23) Novoselov, K. S.; Geim, A. K.; Morozov, S. V.; Jiang, D.; Zhang, Y.; Dubonos, S. V.; Grigorieva, I. V.; Firsov, A. A. Electric field effect in atomically thin carbon films. Science 2004, 306, 666-669.

(24) Butler, S. Z.; Hollen, S. M.; Cao, L.; Cui, Y.; Gupta, J. A.; Gutierrez, H. R.; Heinz, T. F.; Hong, S. S.; Huang, J.; Ismach, A. F.; et al. Progress, challenges, and opportunities in two-dimensional materials beyond graphene. ACS Nano 2013, 7, 2898-2926.

(25) Liu, B.; Abbas, A.; Zhou, C. Two-dimensional semiconductors: From materials preparation to electronic applications. Adv. Electron. Mater. 2017, 3, 1700045.

(26) Turkowski, V.; Din, N. U.; Rahman, T. S. Time-dependent density-functional theory and excitons in bulk and two-dimensional semiconductors. Computation 2017, 5, 39.

(27) Mounet, N.; Gibertini, M.; Schwaller, P.; Campi, D.; Merkys, A.; Marrazzo, A.; Sohier, T.; Castelli, I. E.; Cepellotti, A.; Pizzi, G.; et al. Two-dimensional materials from high-throughput computational exfoliation of experimentally known compounds. Nat. Nanotechnol. 2018, 13, 246-252.

(28) Mak, K. F.; Lee, C.; Hone, J.; Shan, J.; Heinz, T. F. Atomically thin $\mathrm{MoS}_{2}$ : A new direct-gap semiconductor. Phys. Rev. Lett. 2010, 105,136805 .

(29) Jariwala, D.; Sangwan, V. K.; Lauhon, L. J.; Marks, T. J.; Hersam, M. C. Emerging device applications for semiconducting twodimensional transition metal dichalcogenides. ACS Nano 2014, 8, $1102-1120$.

(30) Voshell, A.; Terrones, M.; Rana, M. In Wide bandgap power and energy devices and applications III; Matin, M., Chowdhury, S., Dutta, A. K., Eds.; SPIE, 2018; Vol. 10754; pp $66-83$.

(31) Mueller, T.; Malic, E. Exciton physics and device application of two-dimensional transition metal dichalcogenide semiconductors. $n p j$ 2D Mater. Appl. 2018, 2, 1-12.

(32) Lau, K. W.; Cocchi, C.; Draxl, C. Electronic and optical excitations of two-dimensional $\mathrm{ZrS}_{2}$ and $\mathrm{HfS}_{2}$ and their heterostructure. Phys. Rev. Mater. 2019, 3, 074001.

(33) Edalati-Boostan, S.; Cocchi, C.; Draxl, C. $\mathrm{MoTe}_{2}$ as a natural hyperbolic material across the visible and the ultraviolet region. Phys. Rev. Mater. 2020, 4, 085202.

(34) Liang, Q.; Zhang, Q.; Zhao, X.; Liu, M.; Wee, A. T. Defect engineering of two-dimensional transition-metal dichalcogenides: Applications, challenges, and opportunities. ACS Nano 2021, 15, 2165-2181.

(35) Choi, W.; Choudhary, N.; Han, G. H.; Park, J.; Akinwande, D.; Lee, Y. H. Recent development of two-dimensional transition metal dichalcogenides and their applications. Mater. Today 2017, 20, 116130 . 
(36) Cassabois, G.; Valvin, P.; Gil, B. Hexagonal boron nitride is an indirect bandgap semiconductor. Nat. Photonics 2016, 10, 262-266.

(37) Paleari, F.; Galvani, T.; Amara, H.; Ducastelle, F.; MolinaSánchez, A.; Wirtz, L. Excitons in few-layer hexagonal boron nitride: Davydov splitting and surface localization. 2D Mater. 2018, 5, 045017.

(38) Wickramaratne, D.; Weston, L.; Van de Walle, C. G. Monolayer to bulk properties of hexagonal boron nitride. J. Phys. Chem. C 2018, 122, 25524-25529.

(39) Aggoune, W.; Cocchi, C.; Nabok, D.; Rezouali, K.; Akli Belkhir, M.; Draxl, C. Enhanced light-matter interaction in graphene/h-BN van der Waals heterostructures. J. Phys. Chem. Lett. 2017, 8, 14641471.

(40) Bawari, S.; Pal, S.; Pal, S.; Mondal, J.; Narayanan, T. N. Enhanced photo-electrocatalytic hydrogen generation in graphene/ hBN van der Waals structures. J. Phys. Chem. C 2019, 123, 1724917254.

(41) Li, X.; Mullen, J. T.; Jin, Z.; Borysenko, K. M.; Buongiorno Nardelli, M.; Kim, K. W. Intrinsic electrical transport properties of monolayer silicene and $\mathrm{MoS}_{2}$ from first principles. Phys. Rev. B: Condens. Matter Mater. Phys. 2013, 87, 115418.

(42) Li, H.; Hui-Xia, F.; Meng, S. Silicene: From monolayer to multilayer-A concise review. Chin. Phys. B 2015, 24, 086102.

(43) Houssa, M.; Dimoulas, A.; Molle, A. Silicene: A review of recent experimental and theoretical investigations. J. Phys.: Condens. Matter 2015, 27, 253002.

(44) Chowdhury, S.; Jana, D. A theoretical review on electronic, magnetic and optical properties of silicene. Rep. Prog. Phys. 2016, 79, 126501.

(45) Ramasamy, K.; Sims, H.; Butler, W. H.; Gupta, A. Mono-, few-, and multiple layers of copper antimony sulfide $\left(\mathrm{CuSbS}_{2}\right)$ : A ternary layered sulfide. J. Am. Chem. Soc. 2014, 136, 1587-1598.

(46) Onida, G.; Reining, L.; Rubio, A. Electronic excitations: Density-functional versus many-body Green's-function approaches. Rev. Mod. Phys. 2002, 74, 601-659.

(47) Kresse, G.; Furthmüller, J. Efficiency of ab-initio total energy calculations for metals and semiconductors using a plane-wave basis set. Comput. Mater. Sci. 1996, 6, 15-50.

(48) Blöchl, P. E. Projector augmented-wave method. Phys. Rev. B: Condens. Matter Mater. Phys. 1994, 50, 17953.

(49) Kresse, G.; Joubert, D. From ultrasoft pseudopotentials to the projector augmented-wave method. Phys. Rev. B: Condens. Matter Mater. Phys. 1999, 59, 1758.

(50) Heyd, J.; Scuseria, G. E.; Ernzerhof, M. Hybrid functionals based on a screened Coulomb potential. J. Chem. Phys. 2003, 118, $8207-8215$.

(51) Salpeter, E. E.; Bethe, H. A. A relativistic equation for boundstate problems. Phys. Rev. 1951, 84, 1232.

(52) Sander, T.; Maggio, E.; Kresse, G. Beyond the Tamm-Dancoff approximation for extended systems using exact diagonalization. Phys. Rev. B: Condens. Matter Mater. Phys. 2015, 92, 045209.

(53) Tongay, S.; Zhou, J.; Ataca, C.; Lo, K.; Matthews, T. S.; Li, J.; Grossman, J. C.; Wu, J. Thermally driven crossover from indirect toward direct bandgap in $2 \mathrm{D}$ semiconductors: $\mathrm{MoSe}_{2}$ versus $\mathrm{MoS}_{2}$. Nano Lett. 2012, 12, 5576-5580.

(54) Mudd, G. W.; Molas, M. R.; Chen, X.; Zolyomi, V.; Nogajewski, K.; Kudrynskyi, Z. R.; Kovalyuk, Z. D.; Yusa, G.; Makarovsky, O.; Eaves, L.; Potemski, M.; Fal'ko, V. I.; Patane, A.; et al. The direct-to-indirect band gap crossover in two-dimensional van der Waals indium selenide crystals. Sci. Rep. 2016, 6, 1-10.

(55) Moosakhani, S.; Sabbagh Alvani, A. A.; Mohammadpour, R.; Sainio, J.; Ge, Y.; Hannula, S.-P. Effect of sulfonating agent and ligand chemistry on structural and optical properties of $\mathrm{CuSbS}_{2}$ particles prepared by heat-up method. CrystEngComm 2018, 20, 1527-1535.

(56) Birkett, M.; Savory, C. N.; Rajpalke, M. K.; Linhart, W. M.; Whittles, T. J.; Gibbon, J. T.; Welch, A. W.; Mitrovic, I. Z.; Zakutayev, A.; Scanlon, D. O.; et al. Band gap temperature-dependence and exciton-like state in copper antimony sulphide, $\mathrm{CuSbS}_{2}$. APL Mater. 2018, 6, 084904. 